\title{
Fast magnetization reversal of GdFeCo induced by femtosecond laser pulses
}

\author{
J. Hohlfeld, Th. Gerrits, M. Bilderbeek, and Th. Rasing \\ Research Institute for Materials, University of Nijmegen, Toernooiveld 1, 6525 ED Nijmegen, The Netherlands \\ H. Awano and N. Ohta \\ Tsukuba Research Laboratory, Hitachi Maxell Ltd., 6-20-1 Kinunodai, Yawara, Tsukuba-gun, 300-2496 Japan
}

(Received 13 June 2001; published 5 December 2001)

\begin{abstract}
Pump-pulse induced magnetization reversal of amorphous $\mathrm{Gd}_{23.1} \mathrm{Fe}_{71.9} \mathrm{Co}_{5.0}$ showed a subpicosecond magnetization collapse followed by a slower reversal. The reversal dynamics is well described by the Bloch equation via a reversal time that does not depend on temperature, but strongly decreases with increasing pump fluence. A comparison to data obtained in external saturation field and in remanence opened the way to separate the contributions due to temperature induced effects within single domains from those related to field induced domain formation.
\end{abstract}

DOI: 10.1103/PhysRevB.65.012413

PACS number(s): 72.15.Jf, 78.47.+p, 85.70.Sq

Ultrafast magnetization dynamics is one of the most challenging issues of modern magnetism. To date, two main experimental approaches are followed in order to initiate these dynamics in ferromagnets. The first technique uses short magnetic field pulses to excite a coherent spin precession which changes the direction of the magnetization $M$ on a hundred picosecond time scale. ${ }^{1-7}$ Within the second approach, optical excitations by femtosecond laser pulses are used to affect the magnitude of $M$. Though great care has to be taken to separate real magnetization dynamics from optical artifacts due to spin-independent changes of the electron distributions ${ }^{8,9}$ significant reduction and even erasure of $M$ within less than 2 ps is consistently reported for itinerant ferromagnets. $^{8-15}$ All experiments on femtosecond laser pulse induced magnetization dynamics only concerned the breakdown and recovery of $M$ within a fixed direction and did not address magnetization reversal. However, the speed limit for thermomagnetic writing is of decisive importance for magneto-optical recording, which has become one of the most important technologies for removable storage media. ${ }^{16}$ In particular, the speed of the thermally assisted copying and amplification processes within magnetically amplified magneto-optical systems (MAMMOS) is of high technological interest. ${ }^{17}$

Laser pulse induced magnetization reversal can be sketched as a three-step process: First, the increase of electron and lattice temperature causes a breakdown of $M$ and of coercive field $H_{c}$. Secondly, barrierless magnetization reversal takes place as soon as $H_{c}$ drops below an oppositely directed external field. During the third and last step the sample cools down and all material parameters reach their initial values again. Thus, temperature and field induced effects drive the reversal.

In this paper, we report on femtosecond laser pulse induced magnetization reversal in the presence of an external static field $H_{\mathrm{ext}}$, measured on ferrimagnetic $\alpha-\mathrm{Gd}_{23.1} \mathrm{Fe}_{71.9} \mathrm{Co}_{5.0}$, a typical read-out layer of MAMMOS. We demonstrate that the contributions due to the temperature dynamics within single domains can be separated from those related to transient domain formation. This is achieved by comparison of the results obtained for three different con- figurations of $H_{\text {ext }}$ : (i) $H_{\text {ext }}$ is a saturation field, (ii) $H_{\text {ext }}$ is antiparallel to $M$ and smaller than the $H_{c}$ at room temperature, and (iii) $H_{\mathrm{ext}}=0$. Using this separation we show the crucial importance of transient domain formation for the magnetization dynamics in configurations (ii) and (iii) and show how the reversal time can be tuned over 2 orders of magnitude by the laser fluence. Additional evidence for this result is provided by the shape of hysteresis loops measured at distinct pump-probe delays.

Assuming that the temperature induced magnetization dynamics is known and given by $M_{0}[T(t)]$, the barrierless magnetization reversal induced by an oppositely directed external field follows the Bloch equation

$$
\frac{d M(t)}{d t}=\frac{-M_{0}[T(t)]-M(t)}{\tau},
$$

provided that $M_{0}[T(t)]$ is related to $T(t)$ via the equilibrium magnetization curve. The conventional form of Eq. (1) describes magnetization reversal at fixed temperature $\left(M_{0}\right.$ $=$ const $)$ and is thus not adequate to describe thermomagnetic writing where $M$ and $T$ vary with time. The so called reversal time $\tau$, denoting the material specific response time of $M$ to magnetic fields, is only directly measured at fixed temperature, since the dynamics of thermomagnetic writing depends on $\tau$ and $M_{0}[T(t)]$, i.e., the cooling rate. This means that $\tau$ corresponds to the delay between the recovery and reversal of $M$. Note, that the Landau-Lifshitz-Gilbert equation, widely used to describe the magnetic response to field pulses, predicts no response for antiparallel orientation of $M$ and $H_{\text {ext }}$.

Though Eq. (1) describes transient magnetization reversal irrespective whether it is due to incoherent rotation of $M$ within a single domain state or to the evolution of oppositely directed domains, ${ }^{4}$ the meaning and behavior of $\tau$ differs significantly for both processes. For incoherent rotation within a single domain, $\tau$ represents the electron spin-lattice relaxation time $T_{1}$ which depends on $T$ but not on the effective field $H_{\text {eff }}{ }^{18}$ In contrast, the reversal time related to transient domain evolution is insensitive to temperature but depends on the $H_{\text {eff }}{ }^{4}$ 
In general, all pump-pulse induced magnetization dynamics may be subject to variations of temperature with time and to transient domain formation. Consequently, any experiment must be able to separate these two effects unambiguously. Though the importance of domain formation has been well known for ferrimagnetic magneto-optical media for two decades, ${ }^{19-21}$ it has so far been ignored with respect to ultrafast experiments on ferromagnets. ${ }^{12-14}$ However, the need for external saturation fields to suppress the appearance of multidomain structures was also observed for ferromagnetic nickel. $^{22}$

The experiments were carried out at room temperature in air using $100 \mathrm{fs} / 800 \mathrm{~nm}$ laser pulses generated by a commercial amplified femtosecond laser system (Tsunami, Spitfire, Spectra-Physics). The pulses were sent through a variable attenuation unit before entering a conventional pump-probe setup. The pump-beam was at normal incidence and focused to about $1 \mathrm{~mm}$ [full width at half maximum (FWHM)]. The probe pulses, incident on the sample at $30^{\circ}$ and focussed to a spot size of about $100 \mu \mathrm{m}$, contained $4 \times 10^{3}$ times less energy than the pump pulses and caused negligible self-action. A pump-/probe-spot ratio of 10:1 was chosen to minimize temperature gradients within the probed area. Using a balanced diode scheme as introduced in Ref. 23, we ensured that exclusively the polar Kerr rotation was measured.

A repetitive magnetic field of square wave form was used to guarantee identical initial conditions for each pump-probe pulse pair. It was applied along the easy axis of the sample perpendicular to the surface and phase locked to the laser repetition rate of $20 \mathrm{~Hz}$. Its strength at times between two subsequent pump-probe pulse pairs was chosen to be much larger than the coercive field at room temperature $H_{C}\left(T_{R}\right)$. The application of this saturation field $H_{\text {off }}$ defined the direction of $M$ and erased any magnetization reversal induced by one pump-pulse before the subsequent pulse excited the sample again. Magnitude and sign of the external field present at times when the pump and probe pulses were reflected at the sample $H_{\text {on }}$ will be given below.

The sample was a MAMMOS read-out layer, consisting of a multilayer structure grown by magnetron sputtering on a glass substrate. A $100 \mathrm{~nm}$ thick layer of AlTi on top of the glass substrate served as a heat sink and enhanced the reflectivity of the sample. A $5 \mathrm{~nm}$ thick layer of SiN was used as a buffer layer between the AlTi and the $20 \mathrm{~nm}$ thick film of $\alpha-\mathrm{Gd}_{23.1} \mathrm{Fe}_{71.9} \mathrm{Co}_{5.0}$ which in turn was covered by a $60 \mathrm{~nm}$ thick SiN protection layer. By adding the buffer layer we got identical interfaces at the ferrimagnetic film. The ferrimagnetism of $\alpha-\mathrm{Gd}_{23.1} \mathrm{Fe}_{71.9} \mathrm{Co}_{5.0}$ results from antiferromagnetic coupling of the ferromagnetic rare earth (RE) and transition metal (TM) subsystems. It can be well described in terms of mean field theory that assumes the magnetization of each subsystem to depend on the mean field determined by the magnetization of both subsystems. ${ }^{24,25}$ This coupling leads not only to one common Curie-temperature $T_{C}$ but also to a compensation temperature $T_{\text {comp }}$ at which the RE and TM magnetizations cancel each other. For our sample, $T_{C}$ and $T_{\text {comp }}$ were 532 and $\approx 260 \mathrm{~K}$, respectively. Note that only the magnetization of the TM subsystem is probed by the linear Kerr-effect at $800 \mathrm{~nm} .^{26}$

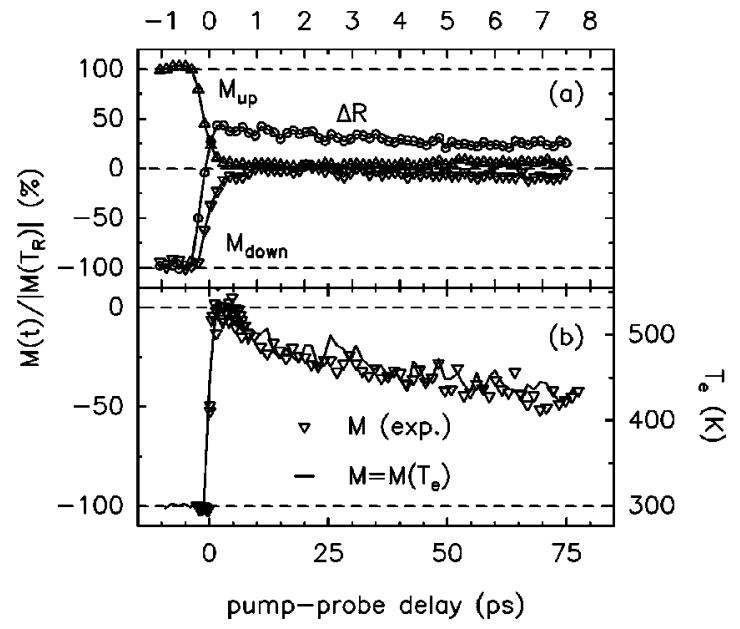

FIG. 1. Magnetization of the TM subsystem of GdFeCo normalized to its magnitude at room temperature as a function of pumpprobe delay. Comparison of (a) the initial magnetization dynamics to simultaneously measured changes of linear reflectivity $(\Delta R)$ monitoring the transient electron temperature $T_{e}$ and of (b) the measured recovery of $M$ at longer delays (symbols) to a theoretical expectation (solid line) which is obtained by transforming the measured $\Delta R(t) \propto T_{e}(t)$ into $M\left[T_{e}(t)\right]$ via the equilibrium magnetization curve.

In order to measure changes of $M$ which were exclusively caused by transient electron- and lattice temperature, we applied a constant saturation field $H_{\text {on }}=H_{\text {on,sat }}=H_{\text {off }}$. Corresponding results obtained for a pump-fluence of $\approx 5.4 \mathrm{~mJ} / \mathrm{cm}^{2}$ are shown in Fig. 1 .

In the upper panel (a) the initial magnetization dynamics, identical for both magnetization directions, is compared to simultaneously measured changes of linear reflectivity which monitor the time evolution of electron temperature $T_{e}$. A very fast and complete breakdown of magnetization during the first picosecond is observed, which is about $500 \mathrm{fs}$ delayed with respect to the increase of $T_{e}$. Measured and calculated dynamics of the subsequent magnetization recovery at longer delay times are compared in the lower panel, Fig. 1(b). The calculation was based on the assumption that $M(t)$ is governed by the electron temperature via the equilibrium magnetization curve. Excellent agreement between data and calculation is found which proves that the recovery of magnetization is solely determined by cooling of the electrons. This result is in line with the behavior of itinerant ferromagnets reported in the literature ${ }^{8-15}$ and justifies the use of Eq. (1) to analyze transient magnetization reversal.

To probe the dynamics of pump-pulse induced magnetization reversal, we choose $H_{\mathrm{on}}=H_{\mathrm{on} \text {,opp }}$ to be antiparallel to the initial magnetization. The magnitude of $H_{\text {on,opp }}$ $\approx 2 / 3 H_{C}\left(T_{\text {room }}\right)$ was adjusted such that it did not affect the magnetization at negative pump-probe delays but that it was large enough to cause significant magnetization reversal within the investigated time range of 800 ps. Since the interpretation of magnetization reversal dynamics in terms of Eq. (1) requires information about both $M(t)$ and $M_{0}(t)$, we periodically switched the external field from $H_{\text {on,opp }}$ (reversal) to $H_{\text {on,sat }}$ (temperature effects) while scanning the pump- 


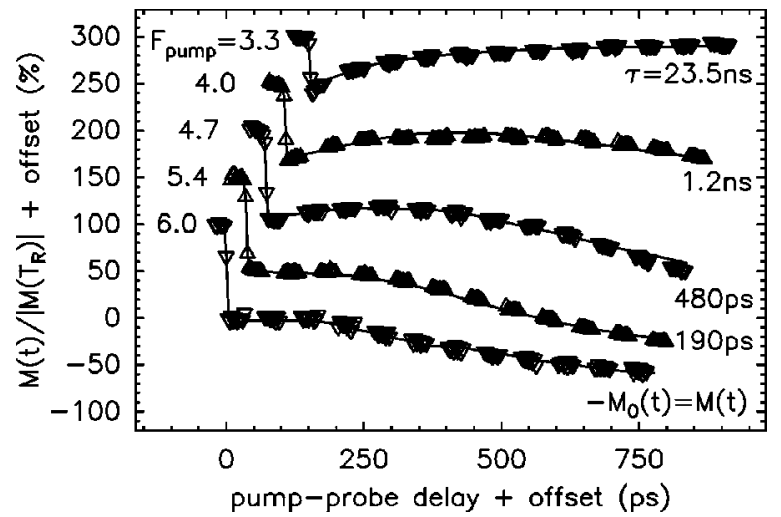

FIG. 2. Transient magnetization reversal dynamics (symbols) measured for distinct pump-fluences, $F_{\text {pump }}$. Solid lines represent best fits of Eq. (1) to the data. Values of $F_{\text {pump }}$ (in units of $\mathrm{mJ} / \mathrm{cm}^{2}$ ) and of the fitted reversal times $\tau$ are given. The data are offset for clarity.

probe delay. In Fig. 2 the data for five pump-fluences are compared to numerical solutions of Eq. (1), which were obtained by taking $M_{0}(t)$ as the negative value of the actual magnetization measured for the external saturation field and treating only the reversal time $\tau$ as a fit parameter.

Each individual data set is excellently fitted by the Bloch equation via one constant value of $\tau \pm 20 \%$ despite the fact that the temperature varies over a large range up to $200 \mathrm{~K}$. This finding proves that not only the temperature induced breakdown and recovery of the TM magnetization but also its reversal dynamics is in line with the behavior of pure ferromagnets, since Eq. (1) does not account for the coupling between the TM and RE moments. The fitted reversal times decrease strongly with increasing pump fluence $F_{\text {pump }}$. A value of $(190 \pm 40) \mathrm{ps}$ is found for $F_{\text {pump }}=5.4 \mathrm{~mJ} / \mathrm{cm}^{2}$, where the Curie temperature is just reached within the probed area, but no delay between the recovery and reversal dynamics is found for a higher fluence of $6.0 \mathrm{~mJ} / \mathrm{cm}^{2}$, where also the surrounding of the probed spot is heated above $T_{C}$. These results show that the surrounding has a significant influence on the reversal dynamics and that the speed of thermomagnetic writing is only limited by the cooling rate of the sample.

Temperature independent reversal times are expected for magnetization reversal by transient domain formation when variations of the effective field are restricted to the first few picoseconds. We checked this prerequisite by measuring hysteresis loops at distinct pump-probe delays. The results, presented in Fig. 3, demonstrate that $H_{C}$ remains indeed zero for pump-probe delays up to 667 ps. Furthermore, the hysteresis loops show a transition from rectangular shape at negative pump-probe delays (reversal within single domain) to continuous changes of $M$ with $H$ at positive delays, which is characteristic for transient domain formation. Combining these observations with the behavior of $\tau$, we conclude that the magnetization reversal is due to nucleation and growth of oppositely directed domains driven by the external field. Regarding the increasing speed of the reversal process with increasing pump fluence we suggest that it is related to an

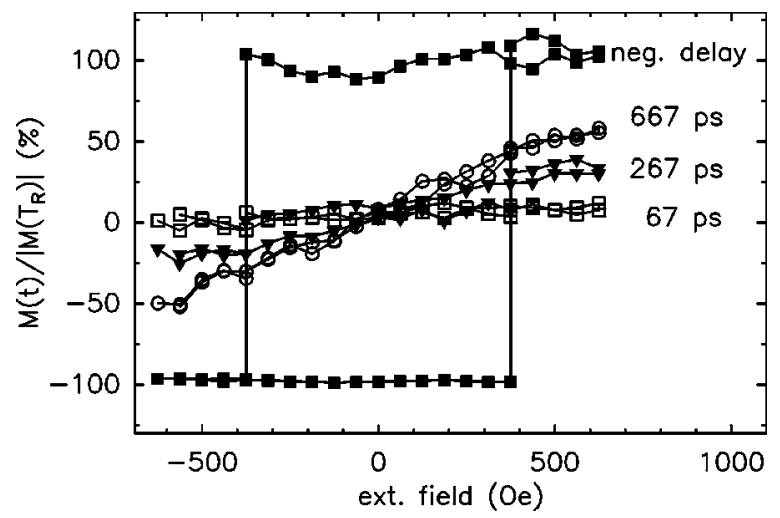

FIG. 3. Hysteresis loops measured for a pump fluence of 5.4 $\mathrm{mJ} / \mathrm{cm}^{2}$ at distinct pump-probe delays given in the figure. The loops demonstrate that the coercive field remains zero for pump-probe delays up to $\approx 670$ ps.

increase in the number of initially created nucleation sites and to a simultaneous decrease of the exchange coupling to the surrounding.

So far, we have discussed pump-pulse induced magnetization dynamics which were controlled by an external field. By comparing these results to data obtained in remanence we can elucidate whether the magnetization dynamics in remanence is determined by temperature effects only ${ }^{13}$ or whether it is also affected by transient domain formation. The data in Fig. 4, obtained for high pump fluence, show that the dynamics in remanence is governed by a convolution of temperature and domain formation effects. This is supported by the perfect agreement of the dynamics observed in remanence and the solid line, representing the sum of the values obtained for $H_{\text {on,sat }}$ and $H_{\text {on,opp }}$. Our results indicate that the delayed breakdown of magnetization observed for thin nickel films by time-resolved photoemission in remanence ${ }^{13}$ could have been caused by analogous effects of domain formation. When this proposition proves to be true, it would reconcile the contradiction to magneto-optical investigations conducted in external saturation fields. ${ }^{8,10}$

In summary, we reported what we believe to be the first

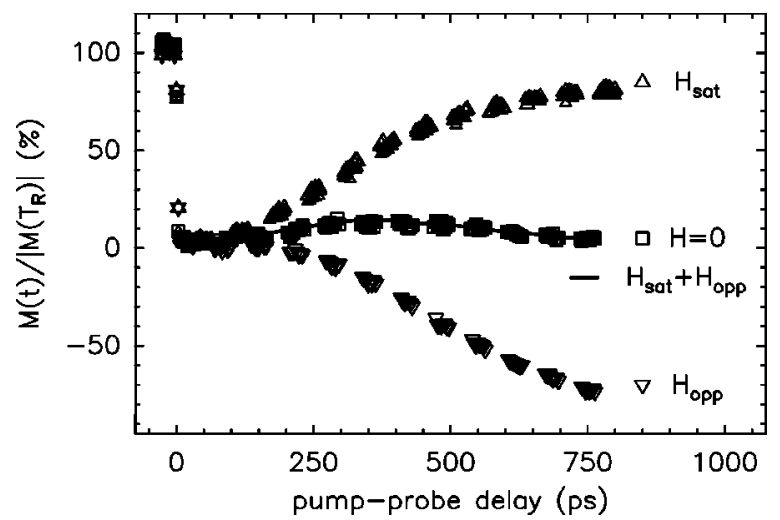

FIG. 4. Comparison of magnetization dynamics measured for $F_{\text {pump }}=5.4 \mathrm{~mJ} / \mathrm{cm}^{2}$ and (1) $H_{\text {on }}=H_{\text {sat }}$, (2) $H_{\text {on }}=H_{\text {opp }}$, and (3) $H_{\text {on }}=0$. The solid line represents the sum of the values obtained for $H_{\text {sat }}$ and $H_{\text {opp }}$. 
observation of femtosecond pump-pulse induced magnetization reversal. For the TM magnetization of ferrimagnetic $\alpha-\mathrm{Gd}_{23.1} \mathrm{Fe}_{71.9} \mathrm{Co}_{5.0}$ the reversal dynamics is perfectly described by the Bloch equation. The corresponding reversal times do not depend on temperature, but decrease strongly with increasing excitation density. Even identical recovery and reversal dynamics were found for the highest pump fluence. However, the observation of a finite reversal time of $(190 \pm 40)$ ps when the temperature within the probed area just exceeds $T_{C}$ indicates significant influence of the colder surrounding. These results point to nanosecond bit access times in MAMMOS, since copying and amplification occur within $\approx 1 \mu \mathrm{m}$ spots at temperatures below $T_{C}$. The behavior of the reversal times as well as the shape of hysteresis loops measured at distinct pump-probe delays provide strong evidence that the magnetization reversal is due to transient domain formation. Regarding the purely temperature induced magnetization dynamics, a fast and complete breakdown of $M$ within the first picosecond is observed, which is about 500 fs delayed with respect to the equilibration of the electron gas. The recovery of $M$ at delay times $>2$ ps is uniquely related to $T_{e}$ via the equilibrium magnetization curve. A comparison of temperature induced dynamics and of fieldinduced magnetization reversal to data obtained for the same high pump-fluence in remanence demonstrates that the dynamics of remanent magnetization cannot be interpreted by temperature dynamics only.

Part of this work was supported by the Stichting Fundamental Onderzoek der Materie (FOM).

${ }^{1}$ T. M. Crawford, P. Kabos, and T. J. Silva, Appl. Phys. Lett. 76, 2113 (2000).

${ }^{2}$ M. Bauer, J. Fassbender, B. Hillebrands, and R. L. Stamps, Phys. Rev. B 61, 3410 (2000).

${ }^{3}$ M. Bauer, R. Lopusnik, J. Fassbender, and B. Hillebrands, Appl. Phys. Lett. 76, 2758 (2000).

${ }^{4}$ N. D. Rizzo, T. J. Silva, and A. B. Kos, Phys. Rev. Lett. 83, 4876 (1999).

${ }^{5}$ C. H. Back et al., Phys. Rev. Lett. 81, 3251 (1998).

${ }^{6}$ R. H. Koch et al., Phys. Rev. Lett. 81, 4512 (1998).

${ }^{7}$ W. K. Hiebert, A. Stankiewicz, and M. R. Freeman, Phys. Rev. Lett. 79, 1134 (1997).

${ }^{8}$ B. Koopmans, M. van Kampen, J. T. Kohlhepp, and W. J. M. de Jonge, Phys. Rev. Lett. 85, 844 (2000).

${ }^{9}$ J. Güdde et al., Phys. Rev. B 59, R6608 (1999).

${ }^{10}$ J. Hohlfeld et al., Appl. Phys. B: Lasers Opt. 68, 505 (1999).

${ }^{11}$ U. Conrad, J. Güdde V. Jähnke, and E. Matthias, Appl. Phys. B: Lasers Opt. 68, 511 (1999)

${ }^{12}$ E. Beaurepaire et al., Phys. Rev. B 58, 12134 (1998).

${ }^{13}$ A. Scholl, L. Baumgarten, R. Jacquemin, and W. Eberhardt, Phys. Rev. Lett. 79, 5146 (1997).

${ }^{14}$ E. Beaurepaire, J.-C. Merle, A. Daunois, and J.-Y. Bigot, Phys.

Rev. Lett. 76, 4250 (1996)

${ }^{15}$ J. Hohlfeld, E. Matthias, R. Knorren, and K.-H. Bennemann, Phys. Rev. Lett. 78, 4861 (1997).

${ }^{16}$ A. K. Zvezdin and V. A. Kotov, Modern Magnetooptics and Magnetooptical Materials (Institute of Physics Publishing, Bristol, 1997), and references therein.

${ }^{17}$ H. Awano et al., Jpn. J. Appl. Phys. 39, 725 (2000).

${ }^{18}$ G. Mitrikas, C. C. Trapalis, and G. Kordas, J. Chem. Phys. 111, 8098 (1999).

${ }^{19}$ M. Mansuripur and G. A. N. Connell, J. Appl. Phys. 55, 3049 (1984).

${ }^{20}$ P. Hansen, J. Appl. Phys. 62, 216 (1987).

${ }^{21}$ M. Schultz and M. Kryder, Appl. Phys. Lett. 54, 1371 (1989).

${ }^{22}$ H. Regensburger, Ph.D. thesis, MPI Halle, 2001; J. Hohlfeld (unpublished).

${ }^{23}$ M. R. Freemann, R. R. Ruf, and R. J. Gambino, IEEE Trans. Magn. 27, 4840 (1991).

${ }^{24}$ R. Hasegawa, J. Appl. Phys. 46, 5263 (1975).

${ }^{25}$ J. F. Herbst and J. J. Croat, J. Appl. Phys. 55, 3023 (1984), and references therein.

${ }^{26}$ T. Katayama, M. Miyazaki, H. Arimune, and T. Shibata, J. Magn. Soc. Jpn. 8, 121 (1984). 